\title{
Susceptibility to atherosclerosis in the apoE*3 Leiden mouse is programmed by intrauterine exposure to maternal under- and overnutrition
}

\author{
Zoe Yates, Elizabeth Tarling, Simon Langley-Evans and Andrew Salter \\ University of Nottingham, Nottingham, UK
}

\begin{abstract}
Epidemiological studies have strongly suggested that the intrauterine environment plays a critical role in determining risk of disease in adulthood $^{(1)}$. Many cohort studies indicate that lower weight at birth, followed by a rapid catch-up growth in childhood is associated with increased late onset of CVD and metabolic syndrome ${ }^{(2)}$. The present study showed that feeding either a low-protein diet or a high-fat 'Western' diet during pregnancy programmes development of atherosclerosis in apoE*3 Leiden (Leiden) mice. Leiden mice carry a naturally-occurring mutation in the human $a p o E$ gene rendering them prone to developing atherosclerosis when fed an atherogenic diet rich in cholesterol ${ }^{(3)}$. Offspring born to wild-type C57B1/6J females mated with Leiden males exposed to a low-protein diet in utero developed more severe atherosclerotic lesions within the aortic arch (2.61-fold greater lesion area compared with similar offspring of females fed a control diet; $P<0.001)$ when fed an atherogenic diet for 12 weeks from weaning. Fetal protein restriction also led to a greater extent of dyslipidaemia, with low-protein-exposed offspring having raised plasma cholesterol $(34 \%$ higher; $P<0.001)$ and plasma TAG $(39 \%$ higher; $P<0.001)$ compared with offspring exposed to a control diet in utero. Analysis of a targeted gene array suggested a role for the LDL receptor, which was found to be underexpressed in the livers of low-protein-exposed mice. Similar programmed repression of sterol regulatory element-binding protein-1c and LDL receptor-related protein-1 suggests disordered lipid metabolism underlies the fetal programming of atherosclerosis seen in this model. Offspring of Leiden females fed a high-fat 'Western' diet during pregnancy developed more severe atherosclerotic lesions within the aortic arch (1.41-fold greater lesion area; $P<0.001)$ when fed an atherogenic diet for 14 weeks from weaning. These data are the first demonstration, in an animal model, that nutrition during gestation can programme CVD as opposed to simply cardiovascular risk. The development of atherosclerosis is therefore dependent on the interaction of genotype, prenatal diet and postnatal diet. This finding highlights the importance of gene-nutrient interactions at very early stages of life in the aetiology of disease.
\end{abstract}

1. Godfrey KM \& Barker DJ (2001) Public Health Nutr 4, 611-624.

2. Eriksson J, Forsen T, Tuomilehto J, Osmond C \& Barker DJ (2001) Int J Obes (Lond) 25, 735-740.

3. Groot PH, van Vlijmen BJ, Benson GM, Hofker MH, Schiffelers R, Vidgeon-Hart M \& Havekes LM (1996) Arterioscler Thromb Vasc Biol 16, 926-933. 